\title{
Impact evaluation of immunisation service integration to nutrition programmes and pediatric outpatient departments of primary health care centers in Rumbek East and Rumbek Centre counties of South Sudan
}

ISRAEL OLUWASEYIDAYO IDRIS ( $\nabla$ ioidris@hneu.net )

Department of Social and Preventive Medicine, V.N Karazin Kharkiv National University, Kharkiv, Ukraine https://orcid.org/0000-0001-8839-8217

\section{Serifu Ayobami Lamidi}

Department of Epidemiology and Medical Statistics, Faculty of Public Health, University of Ibadan, Nigeria

\section{Victor A Ochagu}

Department of Population Health, Faculty of Epidemiology and Population Health, London School of Hygiene and Tropical Medicine, United Kingdom

\section{Janet Tapkigen}

Department of Population Health, Faculty of Epidemiology and Population Health, London School of Hygiene and Tropical Medicine, United Kingdom

\section{Justin Geno Obwoya}

Department of Field Operation and Project Coordination, Health Pooled Fund, South Sudan

\section{Kaja Abbas}

Department of Infectious Disease Epidemiology, Faculty of Epidemiology and Population Health, London School of Hygiene and Tropical Medicine, United Kingdom

\section{Research Article}

Keywords: Immunisation service integration, nutrition sites, children's out-patient department, humanitarian crises, conflict setting, South Sudan

Posted Date: April 19th, 2021

DOI: https://doi.org/10.21203/rs.3.rs-438234/v1

License: (c) (i) This work is licensed under a Creative Commons Attribution 4.0 International License. Read Full License 


\section{Abstract}

\section{Objective:}

To evaluate the impact of immunisation service integration to nutrition programmes and under 5-year-old outpatient departments of primary health care centres in Rumbek East and Rumbek Centre counties of South Sudan.

\section{Design:}

A retrospective intervention study was conducted in selected primary health care centers (PHCCs).

\section{Setting:}

The study was conducted in 3 primary healthcare centres in Rumbek East county and 3 primary healthcare centres in Rumbek Centre county in Lakes state of South Sudan.

\section{Participant:}

We extracted the data for the uptake of pentavalent vaccine (1st, 2nd and 3rd dose) given to children between 6 weeks old to 23 months old from immunisation records for January to June 2019 before immunisation service integration and July 2019 to December 2019 after immunisation service integration on the District Health Information System 2 (DHIS2) website to estimate the immunisation uptake ratio and drop-out rate.

\section{Results:}

The uptake of the first dose of the pentavalent vaccine improved from $61-96 \%(p<0.001)$ after immunisation service integration into the nutrition programmes of the primary health care centers in Rumbek Centre county. The uptake of the second pentavalent dose improved from $37-69 \%(p=0.102)$ and for the third pentavalent dose from $36-62 \%(p<0.001)$, while the drop-out rate reduced from $57-40 \%(p<0.001)$. While in Rumbek East county, the uptake of the first dose of the pentavalent vaccine improved from $55-77 \%(p<0.001)$ after immunisation service integration into the under 5 -year-old pediatric outpatient departments. The uptake of the second dose improved from $36-62 \%(p<0.001)$ and for the third dose from $44-63 \%(p<0.001)$, while the drop-out rate reduced from $40-$ $28 \%(p<0.001)$. Children were $23 \%$ more likely (RR: 1.23 [95\% Cl: $1.01-1.50]$ ) to have been immunised with the first dose of the pentavalent vaccine upon immunisation service integration into the nutrition programmes of primary health care centers of Rumbek Centre county in comparison to integration into under 5-year-old outpatient departments of Rumbek East county.

\section{Conclusion:}

Integration of immunisation service delivery to nutrition sites and children's outpatient departments improved the immunisation coverage and decreased drop-out rate. Stakeholders of the extended programme on immunisation should focus on the sustainability and scale-up of this intervention as it has high potential to improve childhood immunisation coverage and lower the high rates of vaccine preventable deaths among the children in South Sudan.

\section{Key Questions}

\section{Question:}


What is this research focused on exploring, validating, or solving?

- The Immunisation Agenda 2030 highlights integration of immunisation with other essential health services as one of the seven strategic priorities in its framework for action during the decade 2021-2030. In this regard, this research explores the impact of the integration of immunisation with existing vertical OPD and nutrition programs in the primary healthcare settings

\section{Finding:}

What conclusions did this research draw through design, method, and analysis?

- The integration of immunisation services to nutrition programmes and pediatric outpatient departments of primary health care centres in Rumbek East and Rumbek Centre counties of South Sudan improved immunisation coverage and reduced drop-out rates.

- After immunisation service integration into the nutrition programmes of the primary health care centers in Rumbek Centre county, the coverage of the third dose of the pentavalent vaccine improved from $36-62 \%$ while drop-out rate reduced from $57-40 \%$.

- After immunisation service integration into the under 5-year-old pediatric outpatient departments in Rumbek East county, the coverage of the third dose of the pentavalent vaccine improved from $44-63 \%$ while drop-out rate reduced from $40-28 \%$.

\section{Meaning:}

What is the value, meaning and impact of your research? Is there any follow-up study based on this research?

- The beneficial impact of immunisation service integration to nutrition programmes and pediatric outpatient departments of primary health care centres in Rumbek East and Rumbek Centre counties serve as positive examples in improving immunisation coverage and reducing drop-out rates for South Sudan and beyond.

- Immunisation service integration into nutrition programmes and pediatric outpatient departments of primary health care centres need to be sustained in the existing sites and scaled-up to other counties in South Sudan, as logistically as possible.

- As one of the outcomes of this study showed that there is a low immunisation coverage among children above 1-year-old when compared to the younger age children. There is a need to follow-up and identify factors strongly associated with under-immunisation among the older children.

- There is also a need to conduct more studies to explore the impact of the integration of immunisation with other existing essential health services like the Maternal, Newborn, and Child Health (MNCH) program in the primary healthcare settings.

\section{Introduction}

Immunisation is the most cost-effective health intervention against deaths from vaccine preventable diseases, especially in Sub-Saharan Africa $(1,2)$. In South Sudan, vaccine preventable childhood diseases cause the largest mortality among under 5-year-old children with 251 deaths per 1000 live births, 109 deaths per 1000 live births and 99 deaths per 1000 live births in the year 1990, 2010 and 2018 respectively $(3,4)$. While the reduction of under-five mortality rate to 25 deaths or lower per 1000 live births by 2030 is a target of the Sustainable Development Goals as 
well as among the child survival targets of the government of South Sudan, current performance and trends of the health indices indicate that more time beyond 2030 will be required to achieve these targets $(3,5)$.

The health system in South Sudan has been fragile due to the cumulative years of conflict and humanitarian crises which have resulted in poor health outcomes (6-8). However, other countries with similar context including Mozambique $(9,10)$ have made significant health gains $(5,11)$. Globally, infant mortality from vaccine preventable diseases have declined by $24 \%$ between 2010 and 2017 among under 5-year-old children, with more countries receiving polio eradication status from the World Health Organization (WHO) (12). However, the under-five mortality rate in South Sudan has remained the same since 2010 and is significantly higher than the current under-five mortality rate for the African continent $(13,14)$. This is in part due to the low immunisation coverage of vaccines included in the Expanded Programme on Immunization in South Sudan (15), in addition to the introduction of new vaccines being limited $(16,17)$.

The official reported coverage estimates for the third dose of DPT (diphtheria, tetanus toxoids and pertussis) in South Sudan from 2009 to 2019 has been around 50\% (18). This is well below the coverage levels required for herd immunity (19) and $74 \%$ of the country's geopolitical counties are yet to reach $80 \%$ coverage $(19,20)$. Addressing the poor immunisation uptake in South Sudan's fragile environment will play a pivotal role in reversing the trend of vaccine preventable diseases and death in South Sudan. The unmet immunisation coverage is also associated with the sporadic and ongoing insecurity in some states such as the Lakes and Jonglei states (20).

The joint World Health Organization (WHO)/ United Nations International Children's Emergency Fund (UNICEF) Global immunisation vision and strategy (GIVs) aimed at reducing mortality and morbidity from vaccine preventable diseases among under 5-year-old children. The core element of this strategy and the Immunisation Agenda 2030 involves the integration of immunisation services into other health programmes and immunising more people and social groups to improve coverage and uptake $(2,21)$. The Immunisation Agenda 2030 provides a broad global vision and conceptual framework for vaccines and immunisation during the decade of 2021-2030, of which integration of immunisation with essential health services is one of the seven strategic priorities (21). Health service integration has proven effective in improving immunisation uptake in the outpatient department, integrated management of childhood illness, cash transfer health programmes and other integrated community case management programmes $(2,22,23)$.

In South Sudan, the current service delivery model is supported by an existing service integration policy. However, most programmes in immunisation service delivery are implemented vertically and create the integration policypractice gap. While there are broader factors underpinning the choice of vertical or horizontal service delivery, in the context of South Sudan, horizontal (integrated) approach clearly aligns with the South Sudan Health Pooled Fund Phase III priorities of being cost-effective, equitable and locally owned programmes which objective is to reduce maternal and under-five mortality rates in the country (24). This paper therefore believes that the "cost" in costeffectiveness is on the rationale that it is more economically and technically efficient to share resources (particularly human resources) than having them devoted to one programme disjointly, while "effectiveness" is based on the idea that it should produce improvements in certain areas of health for a targeted population (25). In this scenario, integration of health services helps to deal with the health system as a whole. While, in this study, we refer to immunisation service integration as the adoption and assimilation of immunisation services into health system functions considering the health-care system building blocks identified by WHO (26-28). In South Sudan, there is evidence of positive impact of integration on immunisation uptake in nutrition settings (26). However evidence is lacking for under 5-year-old out-patient department settings, and this study addresses this evidence gap. 
The objective of this study is to evaluate the impact of immunisation service integration to nutrition programmes and pediatric outpatient departments of primary health care centres in Rumbek East and Rumbek Centre counties of South Sudan during 2019. Specifically, we evaluated the impact for six months before (January-June 2019) and six months after (July-December 2019) immunisation service integration for the pentavalent vaccine (diphtheria, pertussis, tetanus, hepatitis $\mathrm{B}$, and Haemophilus influenzae type b) into the nutrition programmes of three primary health care centres in Rumbek Centre county and the under 5-year-old out-patient department of three primary health care centres in Rumbek East county.

\section{Methods}

\section{Study design and location}

The South Sudan's healthcare system operates three tiers of healthcare service: community, primary, secondary and tertiary levels $(29,30)(30)$. The community and primary healthcare shares a significant portion of the country's health pooled fund is spent on the community and primary healthcare. The community healthcare system (also called Integrated Community Case Management - iCCM or Boma Health Initiative) operates a delivery mechanism at both community and household level, which is focused on the integrated essential child health care (IECHC) and community based child survival. While the primary healthcare services provide Basic Package of Health Services (BPHS). The BPHS covers preventive, curative, health promotion and managerial activities, which care services are delivered at both the primary healthcare unit and the primary healthcare centre. When compared to the primary healthcare centre, the primary healthcare unit operate only five days per week, eight hour per day and is less staffed with a community healthcare worker, community midwife, a vaccinator and 2 other non-healthcare staff and provides less service which is focused on diagnosis and treatment of common community illnesses, immunisation and therapeutic supplementary feeding programmes with a target to cover 15,000 population. The primary healthcare centre (PHCC) is the first level referral health facility having qualified health professionals including clinical officers, nurses/midwives, pharmacy technician, vaccinators, nutritionist and nutrition assistant offering a wider range of immunisation, basic emergency obstetric and neonatal care (BEmONC), diagnostic, curative, minor surgical services and 24-hour-in-patient care for severe common community illness like severe acute malnutrition , severe malaria,etc. A PHCC is designed and located to cover a population of 25,000 and receives referred cases from 4 PHCUs. It receives cases referred by the health workers of the community healthcare system (also called the Boma health workers) and the primary healthcare unit (30). The immunisation unit of the PHCC is usually a room that is not located in the same building as the nutrition unit, and far $(>30 \mathrm{~m})$ from each other. Similarly, the immunisation unit is usually not close to the out-patient curative consultation department; but sometimes are located in the same building with each other (26).

South Sudan consists of 10 states and 79 counties with a population of 11,062,000 people in 2019. The Lakes State includes Rumbek East and Rumbek Centre counties with a population of 153,550 and 232,752 in 2017 respectively. This was a retrospective intervention study conducted in selected primary health care centers (PHCCs) in Rumbek East county (Aduel PHCC, Pacong PHCC, and Paloc PHCC) and Rumbek Centre county (Amongpiny PHCC, Malual Bab PHCC, and Matangai PHCC) of South Sudan.

\section{Immunisation service integration}

Between July to December 2019, a strategy was implemented to integrate the Expanded Programme on Immunization (EPI) into nutrition programmes in all the primary health care centers in Rumbek Centre and EPI was integrated to under 5-year-old out-patient departments in selected primary health centres in Rumbek East. 
The intervention adopted used both the functional and infrastructural service integration approaches were implemented in a mutualistic relationship between the programmes' service providers with a fully decentralized local planning context which had no much external forces on it $(31,32)$. In this study, we refer to immunisation service integration as the adoption and assimilation of immunisation services into the nutrition service unit and outpatient department of the primary healthcare centre wherein, the static immunisation operational activities were colocated, co-delivered and co-reported in the nutrition service unit and the out-patient department of the primary healthcare centre. Weekly re-orientation/on-job training on quick adoption of the functional approaches and practice of service integration into their daily practice during this integration period were conducted to staff of these two programmes. The practices adopted by these staff included: staff retention practice during the period of intervention, trust between staff, staff-to-staff support, user-centered culture, shared belief and co-ownership between the two programmes' staff, joint-decision-making between the two programmes' staff, regular communication and meetings were regularly monitored. As there are two implementing partners managing the nutrition service programme; and the curative and preventive health services. Programme managers of these two programmes at county level were oriented and accepted to lose territories' ownership of their concerned programs in the immunisation service integration intervention period (32). Lastly, immunisation screening system was also adopted in the nutrition service unit and the out-patient department of the primary healthcare centre, where children between the age of $0-23$ months were screened for zero or under-immunisation before accessing the nutrition services and the curative services; and where applicable, these children were directed to the vaccinators for immunisation. The primary health care centers were regularly monitored for the uptake of $1 \mathrm{st}, 2 \mathrm{nd}$ and $3^{\text {rd }}$ dose of the pentavalent vaccine for 6 months during July-December 2019 (26).

\section{Vaccination schedule}

The pentavalent vaccine is recommended for children at 6, 10, and 14 weeks for the 1 st, 2 nd and 3 rd doses respectively as per the national immunisation schedule in South Sudan. Children who miss out on the recommended vaccination schedule are still offered the pentavalent vaccine upto the age of two years (less than 24 months of age).

\section{District health information system}

The study population includes children between 6 weeks -23 months of age who received one, two or all of the doses of the pentavalent vaccine. All the monthly data of the pentavalent vaccine was solely extracted from the South-Sudan's District Health Information System 2 (DHIS2) website (33), and was used in the analysis. No sampling method was adopted. We strongly believe that the readily available DHIS2 data represents the general population to which the results of the study is applied; in addition to that, it has given our study the power to detect a valid estimate through its large sample size irrespective of socio-economic status of the population. Also, our monitoring and supportive supervision to the vaccinators for proper recording helped in improving the quality of data by ensuring completeness, accuracy and timeliness of data submission.

The records were retrieved on a monthly basis for a 12-month period in 2019 (6 months before and 6 months after intervention) across all the three monitored primary health care centers in each county and was jointly checked with the county health department for data quality and validation. The data was then classified into two groups using the WHO immunisation age groups classification - infant for under- 1 year old age group ( $\geq 6$ weeks and $<12$ months) and toddler for 1 year and above age group ( $\geq 12$ months and $<24$ months), as in this way it is possible to compare the "timely coverage" versus "delayed coverage" respectively. Considering other confounding factors was 
not possible as data from the dhis 2 is strongly restricted and limited to certain variables like age, sex, geographical location - counties, communties.

Immunisation (or vaccine uptake) rate was calculated using the number of children in each targeted age group that received the last recommended pentavalent vaccine dose as the numerator while the estimated number of target population were assumed to be the total number of surviving children in the target group for each vaccine, and was taken as denominator $(34,35)$. This is because the Southern Sudan Centre for Census, Statistics and Evaluation is yet to publish the most accurate and reliable data for the number of surviving children in the local communities of the country. The number of surviving children was obtained by subtracting estimated deaths (using the country's infant mortality rate) from the total live births per month in each county (36). Mathematically, the total number of surviving children at time $t$ as:

Surviving children $=$ Total number of birth delivery - total number of not surviving infants in the considered period Number of surviving children at time t: $P(t)=P(0)-\left(P(0){ }^{*} r\right)$, where:

$P(t)$ is the dead infants after $t$ years

$P(0)$ is the total number of birth delivery at the base month

$r$ is infant mortality rate $(63.7 \%)$

$\mathrm{t}$ is time considered

\section{Comparative effectiveness analysis}

We compared the uptake for the three doses of the pentavalent vaccine before immunisation service integration intervention during January-June 2019 and after immunisation service integration into nutrition sites and children's outpatient departments during July-December 2019, using standard normal distribution test. To address seasonally induced effectiveness bias, we also compared the intervention period (July - December) in 2019 with the same period (July - December) in 2018. Data were stratified by age and immunisation rates were obtained in each age group to adjust for the confounding effect of age. Rate ratios (before and after intervention) were computed to ascertain percentage contribution of intervention programmes in both counties. We calculated rate ratios to estimate the effectiveness of the integration of the Expanded Programme of Immunisation in nutrition settings of Rumbek Centre county in comparison to pediatric outpatient department settings of Rumbek East county. Immunisation drop-out metric was calculated by subtracting the number of pentavalent vaccine 3rd dose uptake from the number of the pentavalent vaccine 1 st dose uptake in each county. The immunisation drop-out metric was divided by the uptake of the pentavalent vaccine $1^{\text {st }}$ dose to estimate the immunisation drop-out rate $(34,35)$. Student t-test was used to compare uptake of immunisation before and after intervention. Standard normal distribution test (Z-test) was used to generate confidence intervals (with 95\% confidence level) for rate ratios. Estimates with p-value less than significance level (5\%) were reported as strongly associated. We conducted our statistical analysis using SPSS version 25.

\section{Ethical approval}

Ethical approval for the study to be conducted and published was granted by the Health Research Ethics Committee of the State Ministry of Health (SMoH) of Lakes State (formerly Western Lakes State), South Sudan (Reference 
Number: MOH/WLS/14/09/2019). The research was carried out in accordance with the principles of the Helsinki declaration.

\section{Patient and Public Involvement statement:}

The immunisation coverage data used in this study is based on children eligible for pentavalent vaccination in the Rumbek East and the Rumbek Centre counties of South Sudan. Children and parents/adult carers were not involved in setting the research question or the outcome measures.

\section{Results}

The monthly uptake of pentavalent vaccine before (January-June 2019) and after (July-December 2019) immunisation service integration into primary health care centers within the nutrition programmes of Rumbek Centre county and pediatric outpatient departments of Rumbek East county are shown in Figure 1.

In the Rumbek Centre county, the uptake rate of the first dose of the pentavalent vaccine improved from $61 \%$ to $96 \%$ after immunisation service integration into the nutrition programmes of the primary health care centers (see Table 1 and Figure 2). The uptake rate of the second dose improved from $37 \%$ to $69 \%$ and for the third dose from $36 \%$ to $62 \%$, while the drop-out rate reduced from $57 \%$ to $40 \%$. In the Rumbek East county, the uptake rate of the first dose of the pentavalent vaccine improved from 55\% to $77 \%$ after immunisation service integration into the under 5 -yearold pediatric outpatient departments of the primary health care centers. The uptake rate of the second dose improved from $36 \%$ to $62 \%$ and for the third dose from $44 \%$ to $63 \%$, while the drop-out rate reduced from $40 \%$ to $28 \%$.

Children were $23 \%$ more likely (rate ratio of 1.23 [1.01 - 1.50]) to have been immunised with the first dose of the pentavalent vaccine upon immunisation service integration into the nutrition programmes of primary health care centers of Rumbek Centre county in comparison to integration into pediatric outpatient departments of Rumbek East county (see Figure 4 and Table 5). But there was no association of relatively higher impact of the immunisation service integration into either of these settings for the second and third doses of the pentavalent vaccine.

Generally, in the two counties, the newest birth cohort had higher vaccination coverage in comparison to previous birth cohorts (see Table 2). In Rumbek Centre county, uptake rate of the first dose of pentavalent vaccine after immunisation service integration into nutrition programmes of the primary health care centers, in comparison to before integration, increased from $55 \%$ to $96 \%(p<0.05)$ and decreased from $7 \%$ to $4 \%(p=0.162)$ among under 1 year-old and 1-year and above children respectively. Similarly, the uptake rate of the second and third doses of the pentavalent vaccine among under 1 -year-old children improved to $65 \%$ and $52 \%$ respectively post-intervention in comparison to $33 \%$ and $32 \%$ respectively pre-intervention; while the uptake rate of the second and third doses of the pentavalent vaccine among 1 -year-old and above children slightly increased to $5 \%$ and $10 \%$ respectively postintervention in comparison to $4 \%$ and $3 \%$ respectively pre-intervention (see Table 2 ). The low coverage rate among children aged 1-year and above can also be seen in the results for Rumbek East county (see Table 2).

In the Rumbek East county, vaccine uptake rate of the first dose of pentavalent vaccine among under 1-year-old children improved to $75 \%$ after immunisation service integration into pediatric outpatient departments of the primary health care centers in comparison to $51 \%$ before integration (see Table 2). Similarly, the uptake rate of the second and third doses of the pentavalent vaccine among under 1 -year-old children improved to $58 \%$ and $58 \%$ respectively post-intervention in comparison to $34 \%$ and $40 \%$ respectively pre-intervention. The uptake rate of the 
pentavalent vaccine improved among children aged 1 year or above after immunisation service integration to $5 \%$ for the third dose post-intervention in comparison to $4 \%$ pre-intervention. The drop-out rates are significantly lower among both under and above 1-year-old children post-intervention in comparison to pre-intervention in Rumbek East county (see Table 2).

To address seasonally induced effectiveness bias, we also compared the impact during the post-intervention period of July - December 2019 with the pre-intervention period of July - December 2018. We observed results similar to the comparative analysis for the period before (January - June 2019) immunisation service integration and after (July - December 2019) immunisation service integration (see Table 3-4 and Figure 3).

\section{Discussion}

While childhood immunisation coverage among under 5-year-old children in low- and middle-income countries is below the global average, the coverage is relatively even lower in South Sudan especially in the conflict affected areas (26). With South Sudan emerging from protracted civil wars, we infer a favourable impact of immunisation service integration into nutrition programmes and pediatric outpatient departments in this study. This highlights a positive way forward to optimise and scale-up the integration of the Expanded Programme on Immunization with other health services in the primary health care centers to improve childhood immunisation coverage in South Sudan. Similar inferences have been highlighted in prior studies on the benefits of integrating the Expanded Programme on Immunization with comprehensive health service delivery and recommending broader adoption in South Sudan $(26,27,37,38)$.

The Expanded Programme on Immunization was initiated in 1974 by the World Health Organization and United Nations International Children's Emergency Fund (39) through a vertical approach which later shifted to an integration approach by integrating immunisation services with other health services within the Integrated Management of Childhood Illness context (40). The reasons for WHO's recommendation for horizontal integration of childhood immunisation with other health programs are improved efficiency and cost savings (41). This stems from a decrease in competition for resources and duplication of the health systems in comparison to vertical programmes. Immunisation service integration also has challenges, such as overburdened healthcare staff, unequal resource allocation, difficult funding mechanism, donor policies, and logistical difficulties (23). Therefore, the benefits inferred in this study for immunisation service integration with nutrition programmes and pediatric outpatient departments in primary health care centers will need to be assessed against the risks imposed by operational logistics and compatibility differences between health programmes for successful integration (28).

The nutrition services in our study were planned to facilitate co-delivery and a shared-information approach was used to ensure that all the services were provided at the nutrition center. Children's nutrition status and growth monitoring are important to mothers and caregivers and hence the drive to attend nutrition services delivery programmes (42). Consequently, nutrition services such as growth monitoring, nutrition counseling and education were placed as core services while immunisation services were also conducted. This highlights the benefits of integrating immunisation services into nutrition programmes to improve the childhood immunisation coverage, as inferred by our study through increased uptake of the pentavalent vaccine.

In the pediatric outpatient departments, recipient-oriented interventions such as recalls and reminders, health education, teaching recipients skills and provider-oriented interventions such as health services audits and feedback mechanism, chart-based or calendar reminders were also provided. While we infer in our study the increased uptake of the pentavalent vaccine, this also highlights the benefits of integrating immunisation services into pediatric 
outpatient departments to improve childhood immunisation coverage. Immunisation service integration into both nutrition programmes and pediatric outpatient departments of primary health care centres have effectively reduced missed opportunities for vaccination.

In the Rumbek Centre county, our inferences on improved immunisation coverage through integration into nutrition programmes are supported by findings by Oladeji et al in a similar study (26) conducted in the Liech state of South Sudan which also found increased childhood immunisation coverage and reduced drop-out rates. The study also highlighted a higher adherence to vaccination among children vaccinated at outpatient therapeutic programme centers in comparison to primary health care centers. Similar supportive evidence have been generated by related studies with a positive impact on nutrition outcomes (43) and that nutrition counseling and education improved coverage of DTP3 vaccination by $68 \%$ (44). Since nutritionists assessed the immunisation status of the children before enrolling them in the nutrition programme, this ensured that missed opportunities for immunisation were assessed and counselled. Also, integration has been successful other way around as well when other interventions such as vitamin A supplementation to immunisation campaigns have improved immunisation coverage (45).

In the Rumbek East county, the improvement in immunisation uptake can be attributed to regular monitoring, motivation, and health education in the pediatric outpatient departments of primary health care centers. Also, mothers/caregivers would like to vaccinate their children in an outpatient clinic where they can access free treatment, and most mothers who deliver in the clinic receive adequate antenatal care which could increase immunisation uptake as well. These findings are corroborated by a systematic review on childhood immunisation interventions in low and middle-income countries, which found that facility-based health education plus redesigned vaccination reminder cards in outpatient departments improved coverage of DTP3 vaccination by $50 \%$ (44). On the other hand, we believe that the impact of the intervention in Rumbek East county was thwarted by a communal conflict between the two major communities - Pacong and Aduel communities from August to September 2019. However, some of the children who missed their immunisation schedule as a result of reduced access and uptake of immunisation and nutrition services due to displacement or fear of mobility were traced and vaccinated using a supplementary immunisation strategy in the month that followed the crisis. This can be seen in the spike of pentavalent 1st dose uptake of Rumbek East county in October 2019 (see Figure 1).

We observed reduction in immunisation drop-out rates after integration in both nutritional programmes and pediatric outpatient departments of primary health care centers. Similar reductions in drop-out rates have been recorded among children attending a pediatric outpatient clinic at Juba Teaching Hospital in 2017 (46) and in a health facility-based study conducted in South Sudan (47). The missed opportunities for immunisation were relatively similar in both Rumbek Centre and Rumbek East counties after immunisation service integration.

Sustaining community-based activities is a major challenge in low- and middle-income countries $(42,48)$. In our study, the nutrition programmes at the primary health care centres of Rumbek Centre county provided nutritional incentives for the community. Provision of incentives especially in community programmes has been shown to be effective in motivating communities, improving effectiveness and impact of the programmes (49).

The distance to access immunisation services is a good predictor of vaccination coverage in low- and middleincome countries. Low vaccination coverage have been reported among caregivers living farther away from the health facilities if no growth monitoring programs and incentives were provided. This finding is consistent with a study in Zambia which showed that immunisation uptake was lower among caregivers living further away from the health facility before a growth monitoring program was introduced into the immunisation services (48). The barrier posed by longer travel distances to access immunisation especially for the second and third doses of the 
pentavalent vaccine could be partially addressed through incentives to attend the primary health care centers in both Rumbek East and Rumbek Centre counties.

Immunisation uptake of the first, second, and third doses of the pentavalent vaccine was higher among under 1year-old children in comparison to children aged above 1-year in both the Rumbek East and Rumbek Centre counties. In West Cameroon, a study showed similar findings where immunisation coverage of the pentavalent vaccine decreased as the children's ages increased (50). Although we did not assess the factors associated with low coverage among children aged above 1-year, other studies have reported limited parental knowledge on child health management, fragile vaccination health services, geographical limitations, economic struggle, mother's level of education, and cultural accessibility as the major reasons for the low uptake $(50,51)$. We hypothesise that the high child care burden of the mothers due to high fertility rates coupled with other household chores, poor community sensitization and internal displacement contributed in part to the low immunisation rates among older children.

Our study has limitations, including the study duration being relatively short in comparison to other studies done on immunisation service integration. Also, a time lagged study will improve the robustness of the results. Taking other

predisposing factors into consideration would be informative (48), such as age and sex of the child, mothers' sociodemographics, lifestyle, and knowledge about immunisation which could be contributing factors for immunisation uptake.

As future directions, cost-effectiveness studies are needed to facilitate decision making and prioritisation of immunisation service integration into nutrition programmes and pediatric outpatient departments while considering the contextual characteristics of workers' training, stakeholders support, vaccine supply chain constraints, and community demand for vaccination.

\section{Conclusion}

In summary, immunisation service integration to nutrition programmes and pediatric outpatient departments of primary health care centres in Rumbek East and Rumbek Centre counties of South Sudan during 2019 has improved the uptake of the pentavalent vaccine and reduced the dropout rates. This positive impact favours policy and practice changes to optimise and scale-up the integration of the Expanded Programme on Immunization with other health services in the primary health care centers to improve childhood immunisation coverage and equity while reducing morbidity and mortality from vaccine preventable infectious diseases among under 5-year-old children in South Sudan.

\section{Declarations}

\section{Availability of data and materials}

The immunisation coverage data used in this study were collected from the DHIS2 with approval from the National Ministry of Health. Children and parents/adult carers were not involved in setting the research question or the outcome measures.

\section{Funding Declaration}

This study was conducted with no fund support from an organization.

\section{Acknowledgements}


We thank the Expanded Program for Immunization Operation team of the State Ministry of Health, Western Lakes State of South Sudan for their involvement and support of the immunisation service integration into the existing nutrition and under 5-year-old pediatric out-patient department programmes. We thank the clinical officers, vaccinators and nutrition workers who participated in the study. We thank Olusola Oladeji and his team for collecting evidence in support of immunisation service integration in South Sudan. KA is supported by the Vaccine Impact Modelling Consortium (OPP1157270).

\section{Declaration of interests}

We declare no competing interests.

\section{Authors' contribution}

IOI conceptualized this study, supervised the implementation of the intervention, organised data management, planned the methodology, analyzed the data and led the report writing. SAL planned the methodology, participated in data management, analyzed the data and participated in report writing. JT participated in data analysis and report writing. KA was involved in study conceptualization, participated in data analysis, and report writing. VO was involved in the study conceptualization, and report writing. JGO was involved in the study conceptualization, supervised the implementation of the intervention, and report writing.

ORCID

IOI (0000-0003-0920-0490); KA (0000-0003-0563-1576)

\section{Bibliography}

1. Jamison DT, Feachem RG, Makgoba MW, Bos ER, Baingana FK, Hofman KJ, et al., editors. Disease and Mortality in Sub-Saharan Africa. 2nd ed. Washington (DC): World Bank; 2006.

2. Wolfson LJ, Gasse F, Lee-Martin S-P, Lydon P, Magan A, Tibouti A, et al. Estimating the costs of achieving the WHO-UNICEF Global Immunization Vision and Strategy, 2006-2015. Bull World Health Organ. 2008 Jan;86(1):27-39.

3. Requejo JH, Bryce J, Barros AJD, Berman P, Bhutta Z, Chopra M, et al. Countdown to 2015 and beyond: fulfilling the health agenda for women and children. Lancet. 2015 Jan 31;385(9966):466-76.

4. Vogt F, Heudtlass P, Guha-Sapir D. Health data in civil conflicts: South Sudan under scrutiny. Centre for Research on the Epidemiology of Disasters (CRED); 2011.

5. GBD 2016 SDG Collaborators. Measuring progress and projecting attainment on the basis of past trends of the health-related Sustainable Development Goals in 188 countries: an analysis from the Global Burden of Disease Study 2016. Lancet. 2017 Sep 16;390(10100):1423-59.

6. Checchi F, Testa A, Warsame A, Quach L, Burns R. Estimates of crisis-attributable mortality in South Sudan, December 2013- April 2018: A statistical analysis. LSHTM; 2018.

7. United Nations Department of Economic and Social Affairs. Least Developed Country Category: South Sudan Profile | Department of Economic and Social Affairs [Internet]. 2018 [cited 2021 Jan 11]. Available from: https://www.un.org/development/desa/dpad/least-developed-country-category-south-sudan.html

8. Lam E, McCarthy A, Brennan M. Vaccine-preventable diseases in humanitarian emergencies among refugee and internally-displaced populations. Hum Vaccin Immunother. 2015;11(11):2627-36. 
9. World Bank. FY21 List of Fragile and Conflict-affected Situations. World Bank; 2020.

10. WHO. World Health Organization | Emergencies [Internet]. 2019 [cited 2021 Jan 11]. Available from: https://www.who.int/emergencies/crises/en/

11. UNICEF, WHO, World Bank Group, United Nations. Levels and trends in child mortality | Report 2019 [Internet]. 2019 [cited 2021 Jan 11]. Available from: https://www.unicef.org/media/60561/file/UN-IGME-child-mortalityreport-2019.pdf

12. WHO. The global vaccine action plan 2011-2020: review and lessons learned: strategic advisory group of experts on immunization. World Health Organization; 2019.

13. WHO. Child mortality and causes of death | World Health Organization [Internet]. 2019 [cited 2021 Jan 11]. Available from: https://www.who.int/data/gho/data/themes/topics/topic-details/GHO/child-mortality-andcauses-of-death

14. UNICEF. South Sudan (SSD) - Demographics, Health \& Infant Mortality - UNICEF Data [Internet]. 2019 [cited 2021 Jan 11]. Available from: https://data.unicef.org/country/ssd/

15. Saint-Victor DS, Omer SB. Vaccine refusal and the endgame: walking the last mile first. Philos Trans R Soc Lond B Biol Sci. 2013 Aug 5;368(1623):20120148.

16. Moszynski P. Refugees in South Sudan to receive pneumococcal vaccine after delays over price. BMJ. 2013 Aug 9;347:f5042.

17. WHO. Global Vaccine Action Plan: Regional vaccine action plans 2016 progress reports. World Health Organization; 2016.

18. WHO. South Sudan: WHO and UNICEF estimates of immunization coverage: 2019 revision. WHO and UNICEF; 2020.

19. Mallory ML, Lindesmith LC, Baric RS. Vaccination-induced herd immunity: Successes and challenges. J Allergy Clin Immunol. 2018 May 24;142(1):64-6.

20. Gavi. Joint Appraisal report 2019, South Sudan [Internet]. 2019 [cited 2020 Dec 1]. Available from: https://www.gavi.org/sites/default/files/document/2020/South\%20Sudan\%20Joint\%20Appraisal\%202019.pdf

21. WHO. Immunization Agenda 2030: A Global Strategy to Leave No One Behind [Internet]. 2020 [cited 2021 Jan 11]. Available from: https://www.who.int/teams/immunization-vaccines-and-biologicals/strategies/ia2030

22. Brits H, Joubert G, Eyman K, De Vink R, Lesaoana K, Makhetha S, et al. An assessment of the integrated nutrition programme for malnourished children aged six months to five years at primary healthcare facilities in Mangaung, Free State, South Africa. South African Family Practice. 2017 Jul 3;59(6):1-5.

23. Wallace A, Dietz V, Cairns KL. Integration of immunization services with other health interventions in the developing world: what works and why? Systematic literature review. Trop Med Int Health. 2009 Jan;14(1):119.

24. UK - Foreign, Commonwealth and Development Office (FCDO). DevTracker Project GB-GOV-1-300427 [Internet]. UK - Foreign, Commonwealth and Development Office (FCDO); 2021 Mar [cited 2021 Apr 18]. Available from: https://devtracker.fcdo.gov.uk/projects/GB-GOV-1-300427

25. World Health Organisation. Module 4 - How to evaluate the programme. [cited 2021 Apr 18]; Available from: https://www.who.int/roadsafety/projects/manuals/alcohol/4-How\%20to.pdf

26. Oladeji O, Campbell P, Jaiswal C, Chamla D, Oladeji B, Ajumara CO, et al. Integrating immunisation services into nutrition sites to improve immunisation status of internally displaced persons' children living in Bentiu protection of civilian site, South Sudan. Pan Afr Med J. 2019 Jan 16;32:28.

Page $13 / 22$ 
27. Clements CJ, Nshimirimanda D, Gasasira A. Using immunization delivery strategies to accelerate progress in Africa towards achieving the Millennium Development Goals. Vaccine. 2008 Apr 7;26(16):1926-33.

28. Palmer AC, Diaz T, Noordam AC, Dalmiya N. Evolution of the child health day strategy for the integrated delivery of child health and nutrition services. Food Nutr Bull. 2013 Dec;34(4):412-9.

29. Roberts B, Guy S, Sondorp E, Lee-Jones L. A basic package of health services for post-conflict countries: implications for sexual and reproductive health services. Reprod Health Matters. 2008 May;16(31):57-64.

30. 30. South Sudan M of H. Ministry of Health, Government of Southern Sudan. Basic Package of Health and Nutrition Services For Southern Sudan - PDF Free Download [Internet]. Ministry of Health, South Sudan; 2009 Jan [cited 2021 Apr 18]. Available from: https://docplayer.net/9884924-Ministry-of-health-government-ofsouthern-sudan-basic-package-of-health-and-nutrition-services-for-southern-sudan.html

31. Armitage GD, Suter E, Oelke ND, Adair CE. Health systems integration: state of the evidence. Int J Integr Care. 2009 Jun 17;9:e82.

32. Mur-Veeman I, Hardy B, Steenbergen M, Wistow G. Development of integrated care in England and the Netherlands: managing across public-private boundaries. Health Policy. 2003 Sep;65(3):227-41.

33. Ministry of Health South Sudan. South Sudan National Health Management Information Systems Datawarehouse [Internet]. 2020 [cited 2020 Aug 1]. Available from: https://www.southsudanhis.org/dhis-webcommons/security/login.action

34. WHO. Analysis and use of health facility data: guidance for immunization programme managers [Internet]. 2018 [cited 2021 Jan 11]. Available from: https://www.who.int/publications/m/item/analysis-and-use-ofhealth-facility-data-guidance-for-immunization-programme-managers

35. MEASURE Evaluation. Immunization coverage rate by vaccine for each vaccine in the national schedule MEASURE Evaluation [Internet]. 2019 [cited 2021 Jan 11]. Available from:

https://www.measureevaluation.org/rbf/indicator-collections/service-use-and-coverage-indicators/dpt3immunization-coverage

36. WHO. GHO | Global Observatory on eHealth - Key Indicators | South Sudan - key indicators [Internet]. 2019 [cited 2021 Jan 11]. Available from: https://apps.who.int/gho/data/node.goe.ki-SSD

37. Centers for Disease Control and Prevention (CDC). Distribution of insecticide-treated bednets during an integrated nationwide immunization campaign-Togo, West Africa, December 2004. MMWR Morb Mortal Wkly Rep. 2005 Oct 7;54(39):994-6.

38. Singh NS, Ataullahjan A, Ndiaye K, Das JK, Wise PH, Altare C, et al. Delivering health interventions to women, children, and adolescents in conflict settings: what have we learned from ten country case studies? Lancet. 2021 Feb 6;397(10273):533-42.

39. World Health Assembly. The Expanded Programme on Immunization: the 1982 resolution by the World Health Assembly. Assignment Child. 1983;(61-62):119-22.

40. 40. Behavioural factors in immunization. World Health Organization; 2000.

41. Mihigo R, Okeibunor J, Masresha B, Mkanda P, Poy A, Zawaira F, et al. IMMUNIZATION AND VACCINE DEVELOPMENT: Progress towards High and Equitable Immunization Coverage in the Africa Region. J Immunol Sci. 2018 Jul 2;Suppl(1):1-9.

42. Igarashi K, Sasaki S, Fujino Y, Tanabe N, Muleya CM, Tambatamba B, et al. The impact of an immunization programme administered through the Growth Monitoring Programme Plus as an alternative way of 
implementing Integrated Management of Childhood Illnesses in urban-slum areas of Lusaka, Zambia. Trans R Soc Trop Med Hyg. 2010 Sep;104(9):577-82.

43. Salam RA, Das JK, Bhutta ZA. Integrating nutrition into health systems: What the evidence advocates. Matern Child Nutr. 2019;15 Suppl 1:e12738.

44. Oyo-Ita A, Wiysonge CS, Oringanje C, Nwachukwu CE, Oduwole O, Meremikwu MM. Interventions for improving coverage of childhood immunisation in low- and middle-income countries. Cochrane Database Syst Rev. 2016 Jul 10;7:CD008145.

45. Pérez-Escamilla R, Engmann C. Integrating nutrition services into health care systems platforms: Where are we and where do we go from here. Matern Child Nutr. 2019;15 Suppl 1:e12743.

46. Malual AC, Jowi Y, Irimu G, Admani B. Missed opportunities for immunization among children attending a Paediatric Outpatient Clinic at Juba Teaching Hospital. South Sudan Medical Journal. 2018.

47. Mbabazi W, Lako AK, Ngemera D, Laku R, Yehia M, Nshakira N. Maiden immunization coverage survey in the republic of South Sudan: a cross-sectional study providing baselines for future performance measurement. Pan Afr Med J. 2013 Nov 23;16:110.

48. Sasaki S, Igarashi K, Fujino Y, Comber AJ, Brunsdon C, Muleya CM, et al. The impact of community-based outreach immunisation services on immunisation coverage with GIS network accessibility analysis in periurban areas, Zambia. J Epidemiol Community Health. 2011 Dec;65(12):1171-8.

49. Haines A, Sanders D, Lehmann U, Rowe AK, Lawn JE, Jan S, et al. Achieving child survival goals: potential contribution of community health workers. Lancet. 2007 Jun 23;369(9579):2121-31.

50. 50. Ateudjieu J, Yakum MN, Goura AP, Tembei AM, Ingrid DK, Bita'a Landry B, et al. EPI immunization coverage, timeliness and dropout rate among children in a West Cameroon health district: a cross sectional study. BMC Public Health. 2020 Feb 13;20(1):228.

51. Russo G, Miglietta A, Pezzotti P, Biguioh RM, Bouting Mayaka G, Sobze MS, et al. Vaccine coverage and determinants of incomplete vaccination in children aged 12-23 months in Dschang, West Region, Cameroon: a cross-sectional survey during a polio outbreak. BMC Public Health. 2015 Jul 10;15(1):630.

\section{Tables}

Table 1: Pentavalent vaccine uptake rate before and after immunisation service integration. Pentavalent vaccine uptake for the three doses and drop-out rates before (January - June 2019) and after (July December 2019) immunisation service integration into the nutrition programmes and pediatric outpatient departments of primary health care centers of Rumbek Centre and Centre and Rumbek East counties respectively. 


\begin{tabular}{|c|c|c|c|c|c|c|}
\hline \multicolumn{7}{|c|}{$\begin{array}{c}\text { Pentavalent vaccine uptake rate before (Jan-June 2019) and after (Jul-Dec 2019) immunisation service } \\
\text { integration }\end{array}$} \\
\hline Rumbek Centre county & Before & After & p-value & RR & Lower & Upper \\
\hline 1st dose & $61.46 \%$ & $95.56 \%$ & $<0.001^{*}$ & 1.55 & 0.741 & 3.262 \\
\hline 2nd dose & $36.52 \%$ & $69.41 \%$ & 0.102 & 1.90 & 0.906 & 3.987 \\
\hline 3rd dose & $35.79 \%$ & $61.83 \%$ & $<0.001^{*}$ & 1.73 & 0.824 & 3.624 \\
\hline Drop-out rate & $57.4 \%$ & $40.00 \%$ & $<0.001^{*}$ & 0.70 & 0.332 & 1.462 \\
\hline Rumbek East county & Before & After & p-value & RR & Lower & Upper \\
\hline 1st dose & $55.00 \%$ & $77.41 \%$ & $<0.001 *$ & 1.41 & 0.671 & 2.953 \\
\hline 2nd dose & $36.24 \%$ & $62.10 \%$ & $<0.001 *$ & 1.71 & 0.817 & 3.595 \\
\hline 3rd dose & $44.00 \%$ & $62.56 \%$ & $<0.001 *$ & 1.42 & 0.678 & 2.983 \\
\hline Drop-out rate & $40.30 \%$ & $28.30 \%$ & $<0.001 *$ & 0.70 & 0.335 & 1.473 \\
\hline
\end{tabular}

Student t-test was used to compare uptake of immunisation before and after intervention. Z-test was used to generate a $95 \%$ confidence interval for rate ratios.

Table 2: Pentavalent vaccine uptake rate before and after immunisation service integration by age. Pentavalent vaccine uptake for the three doses and drop-out rates before (January - June 2019) and after (July - December 2019) immunisation service integration into the nutrition programmes and pediatric outpatient departments of primary health care centers of Rumbek Centre and Rumbek East counties respectively by age groups. 


\begin{tabular}{|c|c|c|c|c|c|c|}
\hline \multicolumn{7}{|c|}{$\begin{array}{l}\text { Pentavalent vaccine uptake rate before (Jan-June 2019) and after (Jul-Dec 2019) immunisation } \\
\text { service integration }\end{array}$} \\
\hline Rumbek Centre county & Before & After & p-value & RR & Lower & Upper \\
\hline 1st dose $(<1$ year $)$ & $54.99 \%$ & $95.56 \%$ & $<0.001 *$ & 1.74 & 0.828 & 3.645 \\
\hline 1st dose ( $\geq 1$ year) & $6.50 \%$ & $4.21 \%$ & 0.162 & 0.65 & 0.309 & 1.359 \\
\hline 2nd dose ( $<1$ year) & $32.53 \%$ & $64.78 \%$ & $<0.001 *$ & 1.99 & 0.949 & 4.178 \\
\hline 2nd dose ( $\geq 1$ year) & $3.99 \%$ & $4.63 \%$ & 0.312 & 1.16 & 0.553 & 2.434 \\
\hline 3rd dose ( $<1$ year) & $32.48 \%$ & $52.37 \%$ & $<0.001 *$ & 1.61 & 0.769 & 3.382 \\
\hline 3rd dose ( $\geq 1$ year) & $3.31 \%$ & $10.09 \%$ & $0.039 *$ & 3.05 & 1.453 & 6.395 \\
\hline Drop-out rate $(<1$ year $)$ & $61.48 \%$ & $47.06 \%$ & $<0.001 *$ & 0.77 & 0.365 & 1.606 \\
\hline Drop-out rate ( $\geq 1$ year) & $54.17 \%$ & $40.63 \%$ & $<0.001 *$ & 0.75 & 0.358 & 1.573 \\
\hline Rumbek East county & Before & After & $\mathrm{p}$-value & $\mathbf{R R}$ & Lower & Upper \\
\hline 1 st dose ( $<1$ year) & $50.79 \%$ & $75.10 \%$ & $<0.001 *$ & 1.48 & 0.705 & 3.102 \\
\hline 1st dose ( $\geq 1$ year) & $4.19 \%$ & $2.30 \%$ & 0.503 & 0.55 & 0.262 & 1.152 \\
\hline 2nd dose ( $<1$ year) & $34.35 \%$ & $57.53 \%$ & $<0.001 *$ & 1.67 & 0.798 & 3.513 \\
\hline 2nd dose ( $\geq 1$ year) & $1.88 \%$ & $4.57 \%$ & 0.092 & 2.43 & 1.159 & 5.099 \\
\hline 3rd dose (<1 year) & $39.73 \%$ & $57.59 \%$ & $<0.001 *$ & 1.45 & 0.691 & 3.041 \\
\hline 3rd dose ( $\geq 1$ year) & $4.24 \%$ & $4.97 \%$ & 0.529 & 1.17 & 0.559 & 2.459 \\
\hline Drop-out rate $(<1$ year) & $38.72 \%$ & $19.59 \%$ & $<0.001 *$ & 0.51 & 0.241 & 1.061 \\
\hline Drop-out rate ( $\geq 1$ year) & $42.11 \%$ & $4.00 \%$ & $<0.001 *$ & 0.09 & 0.045 & 0.199 \\
\hline
\end{tabular}

Student t-test was used to compare uptake of immunisation before and after intervention. The Z-test was used to generate $95 \%$ confidence intervals for rate ratios.

Table 3: Pentavalent vaccine uptake rate before and after immunisation service integration (accounting for seasonality bias). To address seasonally induced effectiveness bias, the intervention impact during the post-intervention period (July - December) in 2019 was compared with the same pre-intervention period (July - December) in 2018. Pentavalent vaccine uptake for the three doses and drop-out rates before (July - December 2018) and after (July - December 2019) immunisation service integration into the nutrition programmes and pediatric outpatient departments of primary health care centers of Rumbek Centre and Rumbek East counties respectively. 


\begin{tabular}{|c|c|c|c|c|c|c|}
\hline \multicolumn{7}{|c|}{$\begin{array}{c}\text { Pentavalent vaccine uptake rate before (July-December 2018) and after (July-December 2019) } \\
\text { immunisation service integration }\end{array}$} \\
\hline Rumbek Centre county & Before & After & $\mathrm{p}$-value & $\mathbf{R R}$ & Lower & Upper \\
\hline 1st dose & $78.19 \%$ & $95.56 \%$ & $<0.001 *$ & 1.22 & 1.083 & 2.564 \\
\hline 2nd dose & $66.27 \%$ & $69.41 \%$ & 0.102 & 1.05 & 0.499 & 2.197 \\
\hline 3rd dose & $46.29 \%$ & $61.83 \%$ & $<0.001^{*}$ & 1.34 & 1.037 & 2.802 \\
\hline Drop-out rate & $54.41 \%$ & $40.00 \%$ & $<0.001 *$ & 0.74 & 0.324 & 0.902 \\
\hline Rumbek East county & Before & After & p-value & $\mathbf{R R}$ & Lower & Upper \\
\hline 1st dose & $63.41 \%$ & $77.41 \%$ & $<0.001^{*}$ & 1.22 & 1.052 & 2.561 \\
\hline 2nd dose & $55.07 \%$ & $62.10 \%$ & $<0.001^{*}$ & 1.13 & 0.538 & 2.366 \\
\hline 3rd dose & $49.24 \%$ & $62.56 \%$ & $<0.001^{*}$ & 1.27 & 1.061 & 2.665 \\
\hline Drop-out rate & $33.49 \%$ & $28.30 \%$ & $<0.001 *$ & 0.85 & 0.403 & 0.973 \\
\hline
\end{tabular}

Student t-test was used to compare uptake of immunisation before and after intervention. Z-test was used to generate a $95 \%$ confidence interval for rate ratios.

Table 4: Pentavalent vaccine uptake before and after immunisation service integration by age (accounting for seasonality bias). To address seasonally induced effectiveness bias, the intervention impact during the post-intervention period (July - December) in 2019 was compared with the same preintervention period (July - December) in 2018. Pentavalent vaccine uptake for the three doses and dropout rates before (July - December 2018) and after (July - December 2019) immunisation service integration into the nutrition programmes and pediatric outpatient departments of primary health care centers of Rumbek Centre and Rumbek East counties respectively by age groups. 


\begin{tabular}{|c|c|c|c|c|c|c|}
\hline \multicolumn{7}{|c|}{$\begin{array}{l}\text { Pentavalent vaccine uptake rate before (July-December 2018) and after (July-December 2019) } \\
\text { immunisation service integration }\end{array}$} \\
\hline Rumbek Centre county & Before & After & p-value & $\mathrm{RR}$ & Lower & Upper \\
\hline 1st dose (<1 year) & $79.05 \%$ & $95.56 \%$ & $<0.001 *$ & 1.21 & 1.056 & 2.536 \\
\hline 1st dose ( $\geq 1$ year) & $4.77 \%$ & $4.21 \%$ & 0.641 & 0.88 & 0.421 & 1.852 \\
\hline 2nd dose (<1 year) & $60.28 \%$ & $64.78 \%$ & 0.134 & 1.07 & 0.512 & 2.254 \\
\hline 2nd dose ( $\geq 1$ year) & $5.98 \%$ & $4.63 \%$ & 0.312 & 0.77 & 0.369 & 1.624 \\
\hline 3rd dose (<1 year) & $32.48 \%$ & $52.37 \%$ & $<0.001 *$ & 1.61 & 1.079 & 3.382 \\
\hline 3rd dose ( $\geq 1$ year) & $5.51 \%$ & $10.09 \%$ & $0.039 *$ & 1.83 & 1.173 & 3.841 \\
\hline Drop-out rate ( $<1$ year) & $54.51 \%$ & $47.06 \%$ & $<0.001 *$ & 0.86 & 0.412 & 0.911 \\
\hline Drop-out rate ( $\geq 1$ year) & $52.28 \%$ & $40.63 \%$ & $<0.001 *$ & 0.78 & 0.370 & 0.863 \\
\hline Rumbek East county & Before & After & p-value & $\mathrm{RR}$ & Lower & Upper \\
\hline 1st dose (<1 year) & $59.45 \%$ & $75.10 \%$ & $<0.001 *$ & 1.26 & 1.054 & 2.483 \\
\hline 1 st dose ( $\geq 1$ year) & $2.29 \%$ & $2.30 \%$ & 0.503 & 1.00 & 0.479 & 2.107 \\
\hline 2 nd dose $(<1$ year $)$ & $50.41 \%$ & $57.53 \%$ & $<0.001^{*}$ & 1.14 & 0.544 & 2.394 \\
\hline 2 nd dose ( $\geq 1$ year) & $7.11 \%$ & $4.57 \%$ & 0.092 & 0.64 & 0.306 & 1.348 \\
\hline 3rd dose (<1 year) & $39.73 \%$ & $57.59 \%$ & $<0.001 *$ & 1.45 & 1.169 & 3.041 \\
\hline 3rd dose ( $\geq 1$ year) & $4.46 \%$ & $4.97 \%$ & 0.529 & 1.11 & 0.531 & 2.338 \\
\hline Drop-out rate $(<1$ year $)$ & $25.52 \%$ & $19.59 \%$ & $<0.001^{*}$ & 0.77 & 0.366 & 1.610 \\
\hline Drop-out rate ( $\geq 1$ year) & $19.11 \%$ & $4.00 \%$ & $<0.001 *$ & 0.21 & 0.100 & 0.439 \\
\hline
\end{tabular}

Student t-test was used to compare uptake of immunisation before and after intervention. The Z-test was used to generate $95 \%$ confidence intervals for rate ratios.

Table 5: Comparative effectiveness of the immunisation service integration into nutrition programmes and pediatric outpatient departments. Effectiveness of the integration of the Expanded Programme of Immunisation into nutrition programmes of Rumbek Centre county in comparison to integration into pediatric outpatient departments of Rumbek East county (using rate ratios and 95\% confidence intervals).

\begin{tabular}{|c|c|}
\hline $\begin{array}{c}\text { Pentavalent vaccine } \\
\text { (3-dose schedule) }\end{array}$ & $\begin{array}{c}\text { Rate ratio } \\
\text { (95\% confidence interval) }\end{array}$ \\
\hline 1st dose & $1.23(1.01-1.50)$ \\
\hline 2nd dose & $1.12(0.57-2.18)$ \\
\hline 3rd dose & $0.99(0.47-2.08)$ \\
\hline
\end{tabular}

Z-test was used to generate $95 \%$ confidence interval for rate ratios 


\section{Figures}
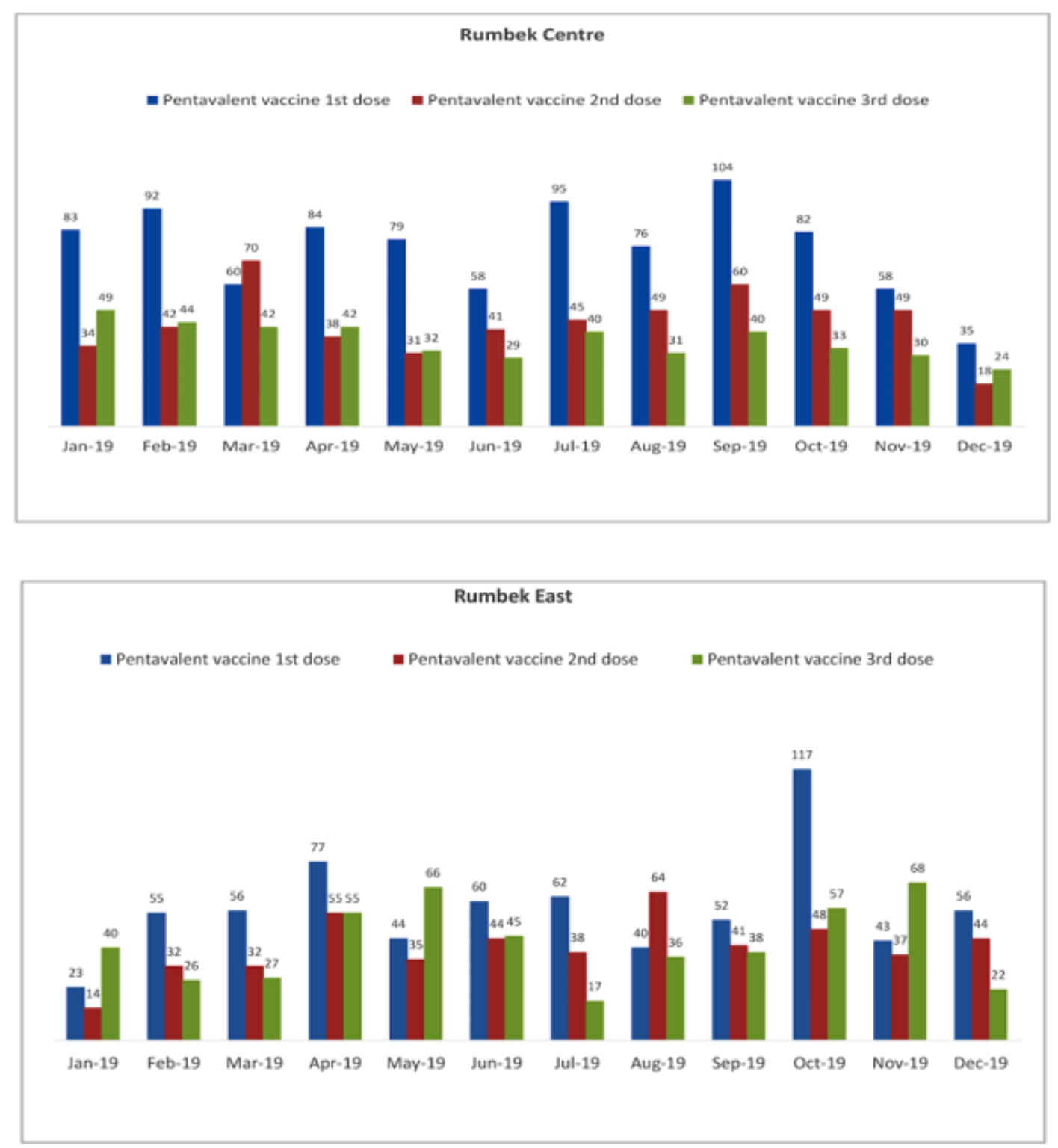

\section{Figure 1}

Pentavalent vaccine uptake before and after immunisation service integration. Monthly uptake of pentavalent vaccine before (January - June 2019) and after (July - December 2019) immunisation service integration into the nutrition programmes and pediatric outpatient departments of primary health care centers of Rumbek Centre and Rumbek East counties respectively. Surviving children eligible for vaccination during the pre-intervention and postintervention periods were 793 and 503 infants respectively in the Rumbek Centre county and 612 and 511 infants respectively in the Rumbek East county. 
Pentavalent vaccine uptake rate before (Jan-June 2019) and after (Jul-Dec 2019) immunisation service integration

Eentavalent vaccine 1st dose (\%)

= Pentavalent vaccine 3 rd dose $(\%)$

$95.56 \%$

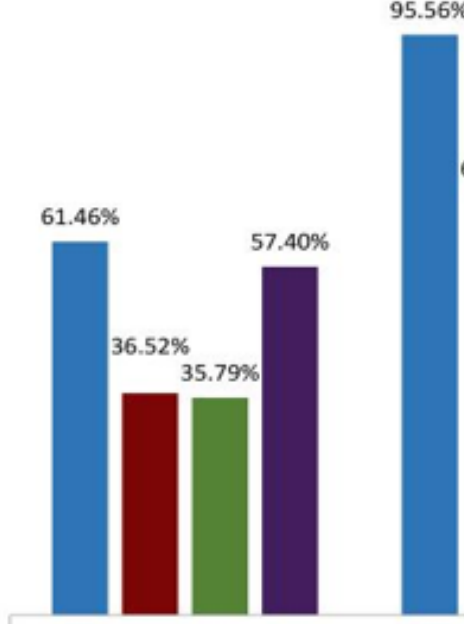

Before

\section{$69.41 \%$}

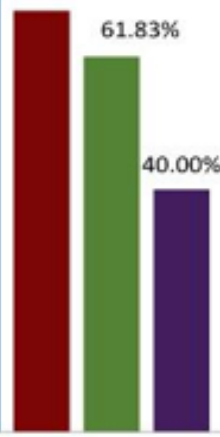

After

- Pentavalent vaccine 2 nd dose (\%)

- Immunization drop-out rate (\%)

$77.41 \%$

$62.10 \% \quad 62.56 \%$

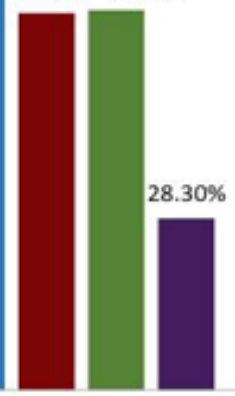

After

Before Rumbek East

Figure 2

Pentavalent vaccine uptake rate before and after immunisation service integration. Pentavalent vaccine uptake rate for the three doses and drop-out rates before (January - June 2019) and after (July - December 2019) immunisation service integration into the nutrition programmes and pediatric outpatient departments of primary health care centers of Rumbek Centre and Rumbek East counties respectively.

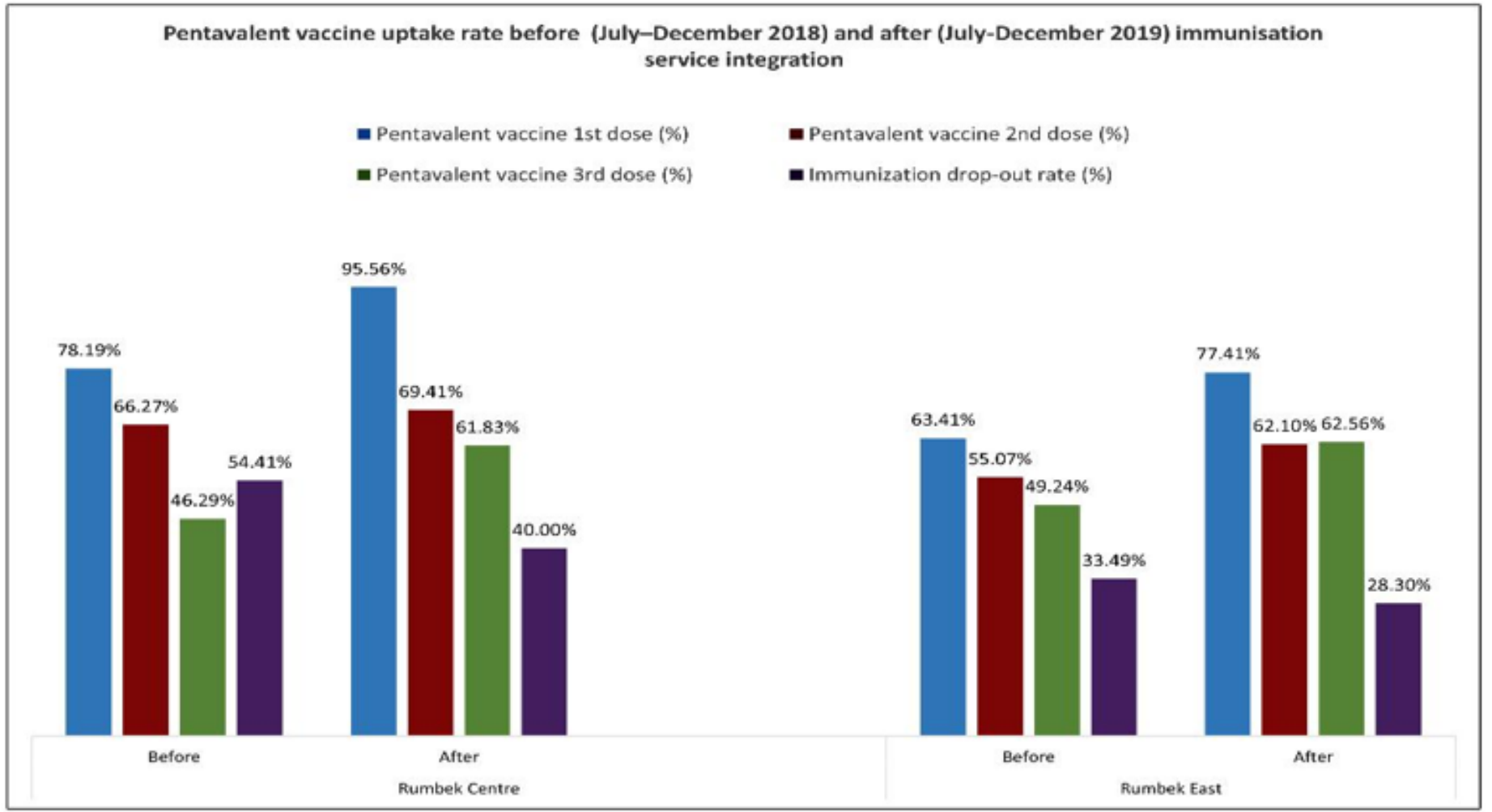


Figure 3

Pentavalent vaccine uptake rate before and after immunisation service integration (accounting for seasonality bias). To address seasonally induced effectiveness bias, pentavalent vaccine uptake rate for the three doses and drop-out rates before (July - December 2018) and after (July - December 2019) immunisation service integration into the nutrition programmes and pediatric outpatient departments of primary health care centers of Rumbek Centre and Rumbek East counties respectively.

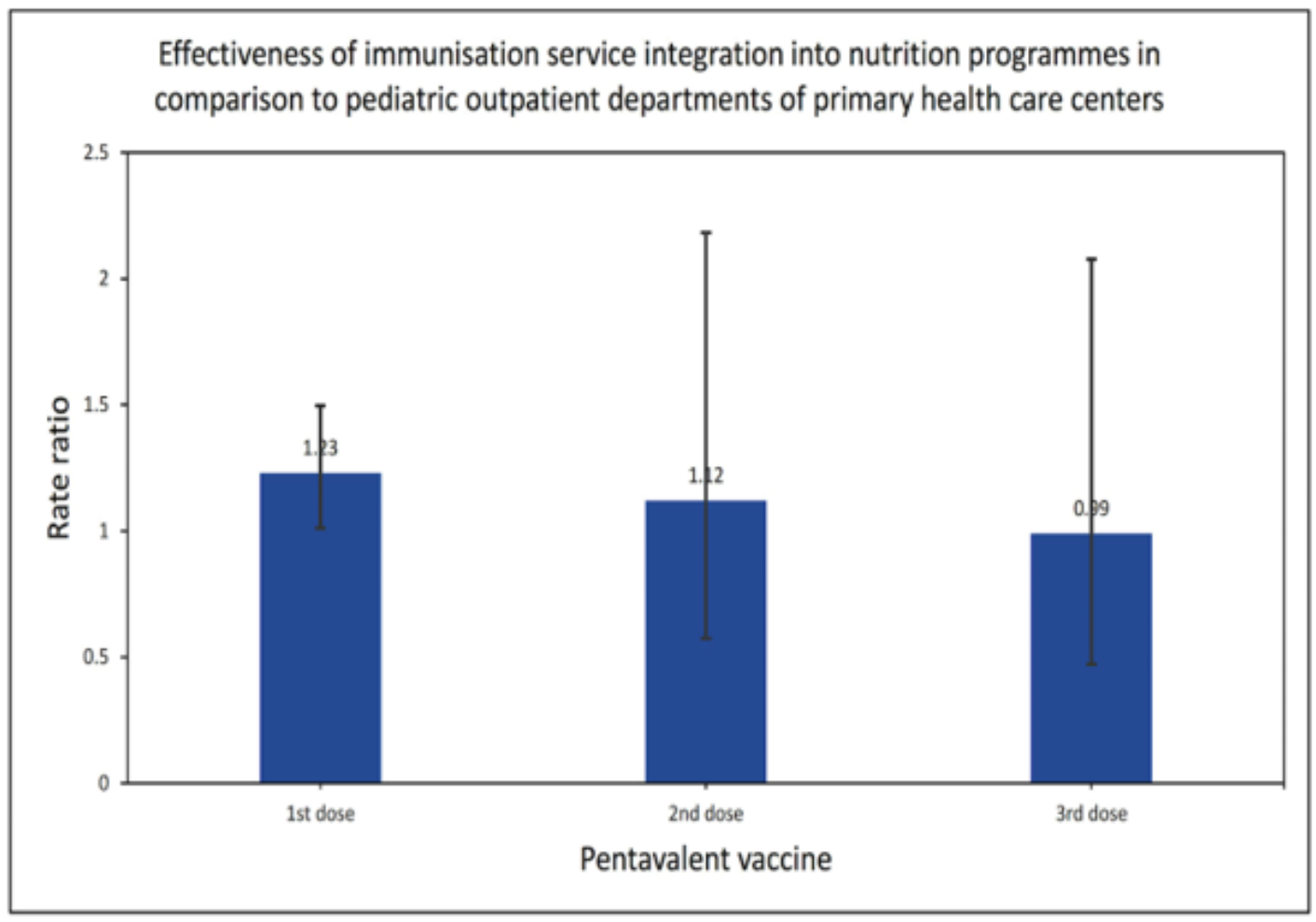

Figure 4

Comparative effectiveness of the immunisation service integration into nutrition programmes and pediatric outpatient departments. Effectiveness of the integration of the Expanded Programme of Immunisation into nutrition programmes of Rumbek Centre county in comparison to integration into pediatric outpatient departments of Rumbek East county (using rate ratios and 95\% confidence intervals). 